\title{
A New Design of Adaptive Fuzzy Hybrid Force/Position Controller for Robot Manipulators
}

\author{
Feng-Yih $\mathrm{Hsu}^{1}$ and Li-Chen $\mathrm{Fu}^{1,2}$ \\ Dept. of Electrical Engineering ${ }^{1}$ \\ Dept. of Computer Science \& Information Engineering ${ }^{2}$ \\ National Taiwan University, Taipei, Taiwan, R.O.C.
}

\begin{abstract}
The major problems of hybrid force/position control arise from uncertainty of the robot manipulators and unknown parameters of the task enviroment. In this paper, a new design method of the hybrid force/position control of the robot manipulators is proposed to solve these problems. The control objective is to track the desired force and position trajectories simultaneously regardless of the unknown parameters of the task enviroment and the existence of the manipulator dynamics, represented as a fuzzy rule-base. The algorithm embedded in the proposed architecture can automatically update the fuzzy rules and, consequently, guarantee the global stability and drive the tracking errors to a neighborhood of zero. The present work is applied to the control of a five degree-of-freedom (DOF) articulated robot manipulator. Simulation results show that the proposed control architecture is featured in fast convergence.
\end{abstract}

\section{Introduction}

Applying the force sensing to the tasks of manipulating objects with robots in contact with surrounding enviroment becomes more and more populatorly adopted. This is mainly due to the technology improvement and the cost-down stimulus of the sensor hardware as well as the rapid advance of the signal processing technology. Typical examples of these tasks are assembly of mechanical parts, deburring, and contour-following operation [1]. These tasks usually require the end effector of robot manipulators to contact the task environment with the desired force and to follow the desired position trajectories. Because of the above-mentioned revolulutionary change, sophisticated methods of simultaneously achieving the two control objectives are thus more feasible.

During the past years, the force control schemes are often referred to as compliant control or hybrid force/position control in the literature [7]-[8]. Generally, the compliant control is trying to simultaneously solve the force control problem and the position control problem in the task frame, and provides reconciliation between the respective solutions of these two problem. Contrarily, the hybrid force/position control is to carefully formulate the task frame so that the associated task space can be easily decomposed into force control subspace and position control subspace, and then achieves the desired respective objectives. In this paper, we will address the problem of the hybrid force/position control.

Since the dynamics of robot manipulators are extremely complex and their surrounding environment is often changing to perform versatile tasks, the nonlinearity terms of the robot manipulators and the parameters of the task environment, e.g., stiffness, can hardly be estimated exactly. Hence, some control which can automatically tune their control laws or update the parameters of the control schemes are necessary and have been proposed such as adaptive control [2]-[3], repetitive control [4] and learning control [5][6]. In this paper, we propose an adaptive robust fuzzy control algorithm to solve the control problems under significant uncertainties in manipulator dynamics and its surrounding enviroment, in which the adaptive part is to on-line update the unknown parameters of the task environment. The overall control scheme not only guarantees the global stability of the closed-loop system but also drives the tracking errors to a neighborhood of zero.

\section{Manipulator Dynamics in Con- straint Coordinate Frame}

When the end effector of a robot manipulators moves in a force-control task along a constraint surface, the constraint coordinate frame attached to the constraint surface are usually selected so as to easily describe the desired force and position trajectories. For simplicity, we assume that the position coordinates of end effector in constraint frame may be represented as a function of its position coordinates in the reference frame, i.e.,

$$
\mathbf{x}_{c}=H(\mathbf{x})
$$

where $\mathbf{x}_{c}=\left[x_{c 1}, \cdots, x_{c_{n}}\right]^{T}(n \leq 6)$ is the position coordinates of the end effector in the constraint frame whereas $\mathrm{x}=\left[x_{1}, \cdots, x_{n}\right]^{T}$ is its position coordinates in the reference frame [5]. Differentiating equation (1), we can derive

$$
\dot{\mathbf{x}}_{c}=\frac{\partial H(\mathbf{x})}{\partial \mathbf{x}} \dot{\mathbf{x}}=R(\mathbf{x}) \dot{\mathbf{x}}
$$


where $R(\mathrm{x}) \in \Re^{n \times n}$ is a transform matrix and is assumed to be nonsingular for $\mathrm{x}$ lying in a compact set in the task space, then there is a one-to-one mapping between $\mathrm{x}_{c}$ and $\mathrm{x}$ in a properly defined compact set due to the implicit function theorem [11]. Besides, denote $J(x)$ as a Jacobian transformation from the joint coordinate frame to the reference coordinate frame. Define a new transformation as $J_{c}(\mathrm{x})=R(\mathrm{x}) J(\mathrm{x})$, then we can derive the dynamics of robot manipulators in the constraint frame as follows. Consider a class of articulated robot arms with $n$ links whose end effectors move along some constraint surfaces. Then, a general dynamic model can be described by

$M\left(\mathrm{x}_{c}\right) \ddot{\mathbf{x}}_{c}+C\left(\mathrm{x}_{c}, \dot{\mathbf{x}}_{c}\right) \dot{\mathbf{x}}_{c}+G\left(\mathrm{x}_{c}\right)+D\left(\mathbf{x}_{c}, \dot{\mathbf{x}}_{c}\right)+\mathbf{f}_{c}=\mathbf{f}$,

where $M_{c}\left(\mathbf{x}_{\mathbf{c}}\right) \in \Re^{n \times n}$ is the inertia matrix, $C\left(\mathbf{x}_{c}, \dot{\mathbf{x}}_{c}\right) \dot{\mathbf{x}}_{c}$ is the vector representing the Coriolis forces, $G\left(\mathbf{x}_{\varepsilon}\right)$ is the vector of gravitational forces, $D\left(\mathbf{x}_{c}, \dot{\mathbf{x}}_{c}\right)$ is the vector of friction forces, $\mathbf{f}_{c} \in \Re^{n}$ is the vector of contact forces, and $\mathbf{f} \in \Re^{n}$ is the $n \times 1$ vector of control input forces. Also, the torque $\tau$ in joint coordinates can be derived as $\tau=J_{c}^{T}(\mathbf{x}) \mathbf{f}$. Generally, when an end effector contacts is in contact with the constraint surface, the desired force trajectories are usually designed for the force elements which are in the directions normal to the surface, and the desired position trajectories are along the tangent directions of the surface, such as deburring tasks or contourfollowing tasks. Hence, normally the force elements along the direction of the desired trajectories are very small and are ignored. Therefore, the vector of the contact forces is defined as $\mathbf{f}_{c}=\left[f_{c 1}, 0, \ldots, 0\right]^{T}$. On the other hand, owing to the flexibility of applying robot manipulators, the manipulator payloads may not be necessarily known prior to a variety of tasks. Because of the frequent change of payloads and of difficult measurements of the friction in the process uncertainties of modeling the manipulators dynamics become inevitable. As a robot, there are some unnegligible deviation between the nominal plant, i.e., $M=\widehat{M}+\Delta M, C=\widehat{C}+\Delta C, G=\widehat{G}+\Delta G$, $D=\widehat{D}+\Delta D$. Our aim is to track both the desired trajectories of force and position, i.e., to force the contact force $f_{c 1}$ and the position vector of the end effector $\mathbf{x}_{c 2}=\left[x_{c 2}, \cdots, x_{c n}\right]^{T}$ to follow the desired trajectories $f_{d_{1}}(t)$ and $\mathbf{x}_{d_{2}}(t)=\left[x_{d 2}, \cdots, x_{d n}\right]^{T}(t)$. Hence, the tracking force error $e_{f}=f_{d_{1}}-f_{c_{1}}$ and the position error vectore $\mathbf{e}_{2}=\left[e_{x 2}, \cdots, e_{x_{n}}\right]^{T}$ are defined. Assume that the robot manipulator is rigid and the deformation of the task environment satisfies the Hook's law, i.e., $f_{c 1}=k_{e}\left(x_{c 1}-x_{s}\right)$ and $f_{d_{1}}=k_{e}\left(x_{d_{1}}-x_{s}\right)$, where $k_{e}$ denotes the stiffness, $x_{s}$ is the position of the constraint surface, and $x_{d 1}$ is the desired position in the force direction. Because the end effector initially contacts the surface with the unknown environmental deformation, the position $x_{s}$ is impossible to be known. Generally, if $k_{e}$ is exactly known, then $e_{f}=$ $k_{e}\left(x_{d 1}-x_{c 1}\right)$ so that $\dot{x}_{d 1}=k_{e}{ }^{-1} \dot{f}_{d 1}$ will transfer the force control problem to the position control problem, i.e., to force $\mathbf{x}_{c}$ to approach $\mathbf{x}_{d d}=\left[x_{d 1}, x_{d 2}, \cdots, x_{d n}\right]^{T}$. However, when $k_{e}$ is unknown and is estimated by $\widehat{k}_{\epsilon}$, the actual desired position $x_{d 1}$ can hardly be obtained. Consequently, apart from the necessity of compensating for the unknown nonlinearity of the robot manipulator, the stiffness of the environment, namely, $k_{e}$, is also required to be identified.

\section{Virtual Adaptive Hybrid Force /Po- sition Control}

Consider a virtual force signal $\widehat{f}_{c 1}$ expressed as $\widehat{f}_{c 1}$ $=\widehat{k}_{e} x_{\varepsilon 1}-\widehat{k_{e} x_{s}}$, where $\widehat{k}_{e}$ and $\widehat{k_{e} x_{s}}$ are the estimate of $k_{e}$ and $k_{e} x_{s}$, respectively. The difference between the actual contact force and virtual force is defined as $e_{p}=f_{c 1}-\widehat{f}_{c 1}$. With $\widehat{k}_{e}$ and $\widehat{k}_{e} x_{s}$ given, a virtual desired position trajectory in the direction of the desired contact force is expressed as $\widehat{x_{d 1}}=\widehat{k}_{e}^{-1}\left(f_{d 1}+\widehat{k_{e} x_{s}}\right)$, where $f_{d_{1}}$ is real desired force trajectory. Our aim is to let $\widehat{x_{d 1}}$ approach the real desired position trajectory $x_{d 1}$ and the position tracking errors $\mathbf{e}_{x_{2}}(t)$ approach zero. Therefore, a robust position controller have to be given in order to ensure the convergence of both the position tracking errors to zero. First, a nominal controller $\widehat{\mathbf{f}}$ based on the computed-torque notion is designed as

$$
\widehat{\mathbf{f}}=\mathbf{f}_{p d}+\widehat{C} \mathbf{x}_{\tau}+\widehat{G}+\widehat{D}+\widehat{M} \dot{\mathbf{x}}_{\tau}+\mathbf{f}_{d}+\mathbf{f}_{e}+\mathbf{e}_{p}
$$

where $\mathrm{f}_{p d}=K \mathbf{s}$ is defined as a PD controller, with $\mathbf{s}=$ $\left[s_{1}, \cdots, s_{n}\right]^{T}=\dot{\mathbf{e}}+\lambda \mathbf{e}$ being defined as a sliding mode vector, $\mathbf{e}=\left[\widehat{e}_{x_{1}}, e_{x_{2}}, \cdots, e_{x n}\right]^{T}$ with $\widehat{e_{x_{1}}}=\widehat{x_{d 1}}-x_{c 1}$, and $\lambda=\operatorname{diag}\left(\lambda_{1}, \cdots, \lambda_{n}\right)$ as a diagonal positive definite matrix; $K=\operatorname{diag}\left(k_{1}, \cdots, k_{n}\right)$ is a positive definite gain matrix; $\mathbf{x}_{r}=\dot{\mathbf{x}}_{d}+\lambda \mathbf{e}$ is an augmented signal vector; $\mathbf{f}_{d}=\left[f_{d 1}, 0, \cdots, 0\right]^{T}$ is the desired force vector; $\mathbf{f}_{e}=\left[\frac{\hat{k}_{e}}{\lambda_{1}} \dot{\hat{e}_{x 1}}, 0 \cdots, 0\right]^{T}$ is an additional compensator. Moreover, the force error $e_{f}$ can be expressed as

$$
\begin{aligned}
e_{f} & =f_{d_{1}}-f_{c 1} \\
& =\widehat{k}_{e}\left(\widehat{x_{d 1}}-x_{c 1}\right)-\left(k_{e}-\widehat{k}_{e}\right) x_{c 1}+\left(k_{e} x_{s}-\widehat{k_{e} x_{s}}\right) \\
& =\widehat{k}_{e}\left(\widehat{x_{d 1}}-x_{c 1}\right)-\left(f_{c 1}-\widehat{f}_{c 1}\right) \\
& =\widehat{k}_{e} \widehat{e}_{x_{1}}-e_{p}
\end{aligned}
$$

This equation induces $\frac{\widehat{k_{e}}}{\lambda_{1}} \dot{\hat{e}_{21}}+e_{f}=\widehat{k_{e}} s_{1}-e_{p}$. Moreover, assume that $0<\sigma_{1}^{B}<k_{e}<\sigma_{1}^{U}$ and $\left|x_{s}\right| \leq \sigma_{2}^{U}$, then let the real controller be given as

$$
\mathbf{f}=\widehat{\mathbf{f}}+\Delta \mathbf{f}
$$

Then, the dynamic equation involving the sliding mode vector $\mathbf{s}$ is given as

$$
M \dot{\mathbf{s}}=-K_{e} \mathbf{s}-C \mathbf{s}+\Delta C \mathbf{s}+h+\Delta M \dot{\mathbf{x}}_{r}-\Delta \mathbf{f},
$$

where $K_{e}=\operatorname{diag}\left(\widehat{k}_{e} / \lambda_{1}+k_{1}, k_{2}, \cdots, k_{n}\right), h(q, \dot{q})=$ $\Delta C \dot{\mathrm{x}}_{c}+\Delta G+\Delta \mathrm{D}$ is an uncertain nonlinear vector, $\mathbf{e}_{p}=\left[e_{p}, 0, \cdots, 0\right]^{T}$. Now, consider the ideal case where the uncertaity of the manipulator dynamics can 
be exactly approximated, so that $\Delta f$ be designed as follows:

$$
\Delta \mathbf{f}=h+\Delta M \dot{\mathbf{x}}_{r}+\Delta C \mathbf{s}
$$

Then equation (7) becomes

$$
\begin{aligned}
M \dot{\mathbf{s}} & =-K_{e} \mathbf{s}-C \mathbf{s}+\Delta C \mathbf{s}+h+\Delta M \dot{\mathbf{x}}_{r}-\Delta \mathbf{f} \\
& =-K_{e} \mathbf{s}-C \mathbf{s}
\end{aligned}
$$

Furthermore, let $\widehat{k}_{e}, \widehat{k_{e} x_{s}}$ be updated as follows:

$$
\begin{aligned}
\dot{\hat{k}}_{e} & =-\dot{\phi_{1}}=r_{1} e_{p} x_{c 1}, \widehat{k}_{e}(0)>0 \\
\dot{k_{e} x_{s}} & =-\dot{\phi_{2}}=-r_{2} e_{p}
\end{aligned}
$$

where $\phi_{1}$ and $\phi_{2}$ are defined as $\phi_{1}=k_{e}-\widehat{k}_{e}$ and $\phi_{2}=k_{e} x_{s}-\widehat{k_{e} x_{s}}$, and $r_{1}$ and $r_{2}$ are some positive constants. Thus, the following proposition will summarize the resulting properties due to the aforementioned control.

Proposition 1 If the control laws are given as in equations (4), (6), (8) and the update law are given by equations (9), (10) then the force and position tracking errors of the robot manipulators system (3) will converge to zeros

Proof:

Define Lyapunov functions $V_{1}(t)$ and $V_{2}(t)$ as $V_{1}(t)=$ $\frac{1}{2} \mathrm{~s}^{T} M \mathrm{~s}$ and $V_{2}(t)=\frac{1}{2 r_{1}}{\phi_{1}}^{2}+\frac{1}{2 r_{2}} \phi_{2}{ }^{2}$, and take their time derivatives, respectively, as

$$
\begin{aligned}
\dot{V}_{1} & =\frac{1}{2} \mathbf{s}^{T}(\dot{M}-2 C) \mathbf{s}-\mathbf{s}^{T} K_{e} \mathbf{s} \\
& =-\mathbf{s}^{T} K_{e} \mathbf{s} \leq 0 \\
\dot{V}_{2} & =\frac{1}{r_{1}} \phi_{1} \dot{\phi}_{1}+\frac{1}{r_{2}} \phi_{2} \dot{\phi}_{2} \\
& =-\left(\phi_{1} x_{c 1}\right)^{2}+2 \phi_{1} \phi_{2} x_{c 1}-\phi_{2}{ }^{2} \\
& =-\left(\phi_{1} x_{c 1}-\phi_{2}\right)^{2} \\
& =-\left(\left(k_{e}-\widehat{k_{e}}\right) x_{c 1}-\left(k_{e} x_{s}-\widehat{k_{e} x_{s}}\right)\right)^{2} \\
& =-\left(e_{p}\right)^{2} \leq 0
\end{aligned}
$$

Then, it is obvious that all signals inside the system are bounded. Howerever, by invoking the Barbalat's lemma [9], it can be easily verified that $s \rightarrow 0$ and $e_{p} \rightarrow 0$, separately, as $t \rightarrow \infty$. This result implies $\mathbf{e} \rightarrow 0$ as $t \rightarrow \infty$ [10]. From equation (5), when $\widehat{e_{x 1}}=0$ and $e_{p}=0$ are obtained, then $e_{f}=0$ is achieved. Hence, as $t \rightarrow \infty, \mathbf{e}_{x 2} \rightarrow 0$ and $e_{f} \rightarrow 0$ are derived. This completes our proof. Remark:

To avoid the singularity in evaluating $\widehat{k}_{e}^{-1}$, the update law is modified as follows. If $\widehat{k}_{e} \leq \sigma_{1}^{B}$,

$$
\begin{aligned}
\dot{\hat{k}_{e}} & =r_{1} \\
\dot{k_{e} x_{s}} & =0 ;
\end{aligned}
$$

otherwise as equations (9), (10) will be assumed. This modification of the update law also guarantees the positivity of $\widehat{k}_{e}$ and, hence, the positive definiteness of $K_{e}$.

Based on Prop. 1, the problem of force control is reduced into the robust position control problem, which is how to design $\Delta f$ properly. Furthermore, we let $\Delta \mathbf{f}=\mathbf{f}_{h}+\mathbf{f}_{s}$, where $\mathbf{f}_{h}$ is designed to approximate the uncertainty of the manipulator dynamics, namely, $h$, and $f_{s}$ is regarded as a robust compensator for the remaining uncertainty. A proper $\Delta f$ is thus defined such that $f_{h}$ approximates $h$ as closely as possible and $\mathbf{f}_{s}$ has a minimum gain. In the next section, we will build a robust controller using the scheme presented in the next section.

\section{Adaptive Fuzzy Hybrid Force / Po- sition Control}

Referring to section 2, the robust control law is given as follows:

$$
\mathbf{f}(t)=\widehat{\mathbf{f}}+\mathbf{f}_{h}^{*}+\mathbf{f}_{s}^{*},
$$

where $\mathbf{f}_{h}^{*}=\widehat{h}$, which is supposed to be an optimal approximation of $h$ in the terms of fuzzy-knowledge representation; $f_{s}^{*}$ is defined as follows:

$$
f_{s k}^{*}=\left(\left|\epsilon_{s k}\right|+U_{M}\left\|\dot{x_{r}}\right\|_{\infty}\right) \operatorname{sgn}\left(s_{k}\right),
$$

where $k$ represents the $k$-th element of vector, $\epsilon_{h}=$ $h-\widehat{h}, \epsilon_{s}=\epsilon_{h}+\Delta C$ s and $U_{M}=\|\Delta M\|_{\infty}$ are given. Here, we will approximate $\mathbf{f}_{h}^{*}$ and $\mathbf{f}_{s}^{*}$ by fuzzy knowledge representation.

\subsection{Fuzzy Knowledge Representation of Uncertainties}

Here our objective is to approximate $\mathbf{f}_{h}^{*}$ and $\mathbf{f}_{s}^{*}$ via fuzzy knowledge representation, where a fuzzy rule-base generally consists of a collection of if-then rules. Now, take $f_{h}^{*}$ as an example to describe this representation. First, let $\mathbf{z}_{h}=\left[\begin{array}{lll}\mathbf{x}_{\mathrm{c}}{ }^{T} & \dot{\mathbf{x}}_{c}{ }^{T}\end{array}\right]^{T}=$ $\left[z_{h 1}, \ldots, z_{h 2 n}\right]^{T}$ be denoted as an input linguistic vector in the discourse universe $U_{\mathbf{z}_{h}}$ and $L_{j}=$ $L_{j}^{1}, L_{j}^{2}, \cdots, L_{j}^{\alpha_{j}}, \cdots, L^{r_{z_{h}}}$ as a family of fuzzy sets associated with the membership functions $\mu_{L_{j} \alpha_{j}}$ 's (see Fig. 1) with respect to the variable $z_{h j}$, where $L_{j}^{\alpha_{j}}$ is a fuzzy set in $L_{j}$. Besides, the cores of the family of fuzzy sets, $L_{j}$, are denoted as $Z_{h_{j}}=$ $\left\{Z_{h_{j}}^{1}, Z_{h_{j}}^{2}, \cdots, Z_{h_{j}}^{\alpha_{j}}, \cdots, Z_{h_{j}}^{r_{h_{j}}}\right\}$, where $Z_{h_{j}}^{\alpha_{j}}$ is a core point satisfying $Z_{h_{j}}^{1}<Z_{h_{j}}^{2}<\cdots<Z_{h_{j}}^{\alpha_{j}}<\cdots<Z_{h_{j}}^{r_{h_{j}}}$ (also see Fig. 1). Let $L$ be defined as a product set of $L=\prod_{i=1} L_{i}$, consisting of the families of fuzzy sets, $L_{i}, i=1, \cdots, 2 n$. Then, an example of the $i$-th fuzzy rule is represented as follows:

$$
R[i]: \text { If } \mathbf{z}_{h} \text { is } L^{\alpha(i)} \text {, then } \mathbf{y} \text { is } Q^{\beta_{(i)}}
$$

where $L^{\alpha_{(i)}} \in L, \alpha_{(i)}=\alpha_{1(i)} \times \cdots \times \alpha_{2 n(i)}$ is a product index associated with the $i$-th rule, $\mathbf{y}=\mathbf{f}_{h}=$ 
$\left[y_{1}, \cdots, y_{n}\right]^{T}$ is denoted as an output linguistic vector, and $Q^{\beta_{(i)}} \in Q, \beta_{(i)}=\beta_{1_{(i)}} \times \cdots \times \beta_{n(i)}$ is another product index associated with the $i$-th rule, $Q$ is another product set of $Q=\prod_{k=1} Q_{k}$, consisting of the families of fuzzy sets, $Q_{k}, k=1, \cdots, n$, where $Q_{k}=\left\{Q_{k}^{1}, Q_{k}^{2}, \cdots, Q_{k}^{\beta_{k}}, \cdots, Q_{k}^{r_{y_{k}}}\right\}$ is denoted as a family of fuzzy sets associated with the membership functions $\mu_{Q_{k}^{\beta_{k}}}$ with respect to the output variable $y_{k}$ with $Q_{k}^{\beta_{k}}$ being a fuzzy set in the family $Q_{k}$. And, let the cores of the family of fuzzy sets, $Q_{k}$, be denoted as $Y_{k}=\left\{Y_{k}^{1}, Y_{k}^{2}, \cdots, Y_{k}^{\beta_{k}}, \cdots, Y_{k}^{\tau_{y_{k}}}\right\}$, where $Y_{k}^{\beta_{k}}$ is a core point satisfying $Y_{k}^{1}<Y_{k}^{2}<\ldots<Y_{k}^{\beta_{k}}<\ldots<Y_{k}^{\tau_{y_{k}}}$. Furthermore, every rule is fired with a weighting function $\rho_{i}\left(\mathbf{z}_{h}\right)$, which is determined by membership functions and a compositional operator. Hence, $\rho_{i}\left(\boldsymbol{z}_{h}\right)$ can be expressed as follows:

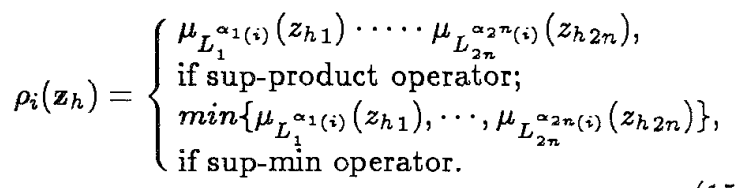

where $\mu_{L_{j}}^{\alpha_{j(i)}}\left(z_{h_{j}}\right)$ is denoted as a membership function, which is a positive function with $\mu_{z_{h_{j}}^{\alpha_{j(i)}}}\left(z_{h_{j}}\right) \leq 1$ and reaches the maximum value when $z_{h j}=Z_{h_{j}}^{\alpha_{j(i)}}$ as shown Fig. 1. In expression (15), the compositional operator is selected to be either the sup-product or the sup-min operator. Finally, using defuzzification function, the output variable $y_{k}$ is expressed as follows:

$$
y_{k}=\frac{\sum_{i=1}^{R_{h}} D_{k i}\left(\rho_{i}\left(\mathbf{z}_{h}\right)\right) Y_{k}^{\beta_{k(i)}}}{\sum_{i=1}^{R_{h}} D_{k i}\left(\rho_{i}\left(\mathbf{z}_{h}\right)\right)},
$$

where $D_{k i}\left(\rho_{i}\left(\mathbf{z}_{h}\right)\right)$ is denoted as a defuzzification function with the core point $Y_{k}^{\beta_{k(i)}}$ for the $i$-th rule commonly expressed as follows:

$$
D_{k_{i}}\left(\rho_{i}\left(\mathbf{z}_{h}\right)\right)=\left\{\begin{array}{l}
\rho_{i}\left(\mathbf{z}_{h}\right), \text { if center-average-defuzzifier; } \\
\int_{-\infty}^{\infty} \mu_{Q^{\beta_{k(i)}}}\left(y_{k}\right) d y_{k}-\int_{y_{k}^{*}}^{y_{k}^{*}}\left[\mu_{Q^{\boldsymbol{\beta}_{k(i)}}}\left(y_{k}\right)\right. \\
\left.-\rho_{i}\left(\mathbf{z}_{h}\right)\right] d y_{k}, \\
\text { if center-of-area defuzzifier; }
\end{array}\right.
$$

where $y_{k}^{-*}$ and $y_{k}^{*}$ are solution values of $y_{k}$ satisfying $\rho_{i}\left(\mathbf{z}_{h}\right)=\mu_{Q_{k} \boldsymbol{\beta}_{k(i)}}\left(y_{k}\right)$ with $y_{k}^{*} \geq y_{k}^{-*}$ given the values $\mathbf{z}_{h}$ with $R_{h}$ being the total number of rules generally equal to $r_{h_{1}} \times r_{h_{2}} \times \cdots \times r_{z_{h 2} 2}$. Then, we denote a fuzzy basis function $\xi_{h_{k i}}\left(\mathbf{z}_{h}\right)$ expressed as follows:

$$
\xi_{h_{k i}}\left(\mathbf{z}_{h}\right)=\frac{D_{k_{i}}\left(\rho_{i}\left(\mathbf{z}_{h}\right)\right)}{\sum_{i=1}^{R_{h}} D_{k_{i}}\left(\rho_{i}\left(\mathbf{z}_{h}\right)\right)}
$$

so that equation (16) can be rewritten as:

$$
y_{k}=\sum_{i=1}^{R_{h}} Y_{k}^{\beta_{h(i)}} \xi_{h_{k i}}\left(\mathbf{z}_{h}\right)=\Theta_{h k}{ }^{T} \xi_{h_{k}}\left(\mathbf{z}_{h}\right)
$$

where $\Theta_{h_{k}}=\left[Y_{k}^{\beta_{k_{(1)}}}, Y_{k}^{\beta_{k_{(2)}}}, \cdots, Y_{k}^{\beta_{k_{\left(R_{h}\right)}}}\right]^{T}$ is regarded as a parameter vector and $\xi_{h_{k}}=\left[\xi_{h_{k 1}}, \xi_{h_{k 2}}\right.$ $\left., \cdots, \xi_{h_{k} R_{h}}\right]^{T}$ is regarded as a regressor vector. For simple in notations, we define a product operator $\otimes$ for two matrices $\Theta \in \Re^{n \times p}, \xi \in \Re^{p \times n}$ as

$$
\Theta \otimes \xi=\left[\Theta_{1}^{T} \xi_{1}, \cdots, \Theta_{k}{ }^{T} \xi_{k}, \cdots, \Theta_{n}{ }^{T} \xi_{n}\right]^{T}
$$

where $\Theta_{k}$ is the $k$-th row vector of $\Theta$ and $\xi_{k}$ is the $k$ th column vector of $\xi$, respectively. Hence, we denote the matrices $\Theta_{h} \in \Re^{n \times R_{h}}, \xi_{h} \in \Re^{R_{h} \times n}$ with the $k$-th row vector denoted as $\Theta_{h_{k}}$ and the $k$-th column vector denoted as $\xi_{h_{k}}$, respectively. Then the output vector $\mathrm{y}$ is expressed as

$$
\mathbf{y}=\Theta_{h} \otimes \xi_{h} .
$$

Apparently, when $R_{h}$ is large, the computation time and memory-space usage must be considered in real implementation. As a result, we focus our attentions on computation time first, then the number of the fired rules and that of the total rules should be clearly distinguished. First, collect the indices of the fired rules as the following set:

$$
I=\left\{i: \rho_{i}\left(\mathbf{z}_{h}\right)>0\right\},
$$

where $\rho_{i}\left(\mathbf{z}_{h}\right)$ is the weighting function of the $i$-th fired rules. Our aim is to figure out that the number of $I$, $\# n(I)$, is less than the number of total rule $R_{h}$ during firing the fuzzy rules along with the expression (16) satisfied.

At the beginning, define the domain set of the total rules with respect to $\mathbf{z}_{h}$ as

$$
E=\left\{\mathbf{z}_{h}: Z_{h_{j}}^{1} \leq z_{h j} \leq Z_{h_{j}}^{r_{h_{j}}}, j=1, \cdots, 2 n\right\}
$$

Therefore, if $\mathbf{z}_{h}$ falls into $E$, then the fuzzy rules are fired to compensate the unknown functions. Partition this domain set into the finite $2 n-$ cells, which are defined as

$$
E_{\alpha}=\left\{\mathbf{z}_{h}: Z_{h_{j}}^{\alpha_{j}} \leq z_{h_{j}} \leq Z_{h_{j}}^{\alpha_{j}+1}, j=1, \cdots, 2 n\right\},
$$

where $\alpha=\alpha_{1} \times \cdots \times \alpha_{j} \times \cdots \times \alpha_{2 n}$ is a product index and satisfies $Z_{h}^{\alpha} \in Z_{h}, Z_{h_{j}}^{\alpha_{j}}<Z_{h_{j}}^{r_{h_{j}}}$. To union the all colection of $E_{\alpha}$, we can obtain $E=\bigcup_{Z_{h}^{\alpha} \in Z_{h}} E_{\alpha}$.

A $\delta-b o x$ is defined as an equivalent of $E_{\alpha}$,

$$
\begin{aligned}
\Omega_{\alpha}\left(Z_{h}^{\alpha}, \delta\left(\mathbf{z}_{h}\right)\right) & =\left\{\mathbf{z}_{h}: Z_{h_{j}}^{\alpha_{j}} \leq z_{h_{j}} \leq Z_{h_{j}}^{\alpha_{j}}+\delta_{j}\left(z_{h}\right)\right\}, \\
& =E_{\alpha}
\end{aligned}
$$

where $j=1, \cdots, 2 n, \delta\left(\mathbf{z}_{h}\right) \in \Re^{2 n \times 1}$ and the $j$-th element of $\delta\left(\mathbf{z}_{h}\right)$ is defined as $\delta_{j}\left(z_{h}\right)=Z_{h_{j}}^{\alpha_{j}+1}-Z_{h_{j}}^{\alpha_{j}}$. Define the set of all corner points of $\delta$-box $\Omega_{\alpha}$ as $P_{\alpha}$ $=\left\{Z^{c\left(z_{h}\right)}: c\left(z_{h}\right) \in \prod_{j=1}^{2 n}\left\{\alpha_{j}, \alpha_{j}+1\right\}\right\}$. Hence the number of $P_{\alpha}, \# n\left(P_{\alpha}\right)$ is equal to $2^{2 n}$.

Consider the membership function (Fig. 1):

$$
\mu_{L_{j}}^{\alpha_{j}}\left(z_{h_{j}}\right)= \begin{cases}1, & \text { as } z_{h j}=Z_{h_{j}}^{\alpha_{j}} ; \\ 0, & \text { as } z_{h_{j}} \geq Z_{h_{j}}^{\alpha_{j}+1} \text { or } z_{h_{j}} \leq Z_{h_{j}}^{\alpha_{j}-1} ; \\ x_{j}^{\alpha_{j}}, & x_{j}^{\alpha_{j}} \in(0,1), \text { otherwise, }\end{cases}
$$


for $\alpha_{j}=1,2, \cdots, l_{j}$ and $j=1,2, \cdots, 2 n$, then the following proposition is given:

Proposition 2 If the membership functions are given as eq. (23) and $\mathrm{z}_{h}$ is the linguistic vector, $\mathrm{z}_{h} \in E$, then exists a $\delta$-box, $\Omega_{\alpha}\left(Z_{h}^{\alpha}, \delta\left(\mathbf{z}_{h}\right)\right)$ such that $\mathbf{z}_{h} \in$ $\Omega_{\alpha}\left(Z_{h}^{\alpha}, \delta\left(z_{h}\right)\right)$, and $\# n(I) \leq \# n\left(P_{\alpha}\right)$, where $\# n(I)$ and \#n(P $\left.P_{\alpha}\right)$ are the number of fired fuzzy rules and that of corner points of $\delta$-box, respectively.

Hence, the computation time is drastically reduced, especially, as $R_{h}$ is very large.

\subsection{Adaptive Robust Fuzzy Hybrid Con- trol}

Based on the fuzzy knowledge representation in subsection 4.1, we will use this representation to approximate the robust compensator $f_{s}^{*}$. Besides, the parameter matrices are also to be determined by adaptive scheme. Let $\mathbf{z}_{s}=\left[\mathbf{x}_{c}{ }^{T} \dot{\mathbf{x}}_{c}{ }^{T} \mathbf{s}^{T}\right]^{T}=\left[z_{s 1}, \cdots, z_{s k}\right.$, $\left.\cdots, z_{s 3 n}\right]^{T}$ be denoted as another input linguistic vector with respect to $\mathbf{f}_{s}$ in the discourse universe $U_{\mathbf{z}_{s}}$, and the cores of the family of fuzzy sets with respect to $z_{s k}$ are denoted as

$$
Z_{s_{k}}=\left\{Z_{s_{k}}^{1}, Z_{s_{k}}^{2}, \cdots, Z_{s_{k}}^{r_{s_{k}}}\right\} \text {, where }
$$

$Z_{k}^{1}<Z_{s_{s_{k}}}^{2}<\cdots<Z_{s_{k}}^{r_{s k}}$ and $k=1,2, \cdots, 3 n$.

Let $\mathbf{f}_{s}$ is denoted as $\mathbf{f}_{s}=\left[f_{s_{1}}, \cdots, f_{s_{k}}, \cdots, f_{s_{n}}\right]^{T}$, where $f_{s k}$ be expressed as follows:

$$
f_{s_{k}}=\Theta_{s k}{ }^{T} \xi_{s_{k}}+\Theta_{M_{k}}{ }^{T} \xi_{M_{k}}\left\|\dot{x_{r}}\right\|_{\infty}
$$

where $\Theta_{s k}$ and $\Theta_{M k}$ are denoted as parameter vectors, $\xi_{s k}, \xi_{M_{k}}$ are denoted as regressor vectors as in equation $(19)$, and $R_{s}$ denotes the total number of rules for $\mathrm{f}_{s}$. Hence the robust compensator vector $\mathrm{f}_{s}$ can be expressed as follows:

$$
\mathbf{f}_{s}=\Theta_{s} \otimes \xi_{s}+\Theta_{M} \otimes \xi_{M}\left\|\dot{x}_{r}\right\|_{\infty},
$$

where the matrices $\Theta_{s}, \Theta_{M} \in \Re^{n \times R_{s}}$ consit of the $k$-th row vectors $\Theta_{s k}, \Theta_{M k}$, respectively, and $\xi_{s}, \xi_{M}$ $\in \Re^{R_{s} \times n}$ consist of the $k$-th column vector $\xi_{s k}, \xi_{M k}$ for $k=1, \cdots, n$, respectively. Our goal is to design the optimal parameter matrices such that the controlled system has minimal tracking errors and robust features. At the beginning, we must prove that bounds on approximation errors depend on our desigri. The the $k$-th column vectors of optimal parameter matrices $\Theta_{h}^{*} . \Theta_{s}^{*}$ and $\Theta_{M}^{*}$ are defined as follows:

$$
\begin{array}{r}
\Theta_{h k}^{*}=\arg \min \left[\sup _{z_{h} \in E}\left|\Theta_{h k}^{T} \xi_{h_{k}}-f_{h k}^{*}\right|\right](26) \\
\Theta_{s k}^{*}=\arg \min \left[\sup _{z_{s} \in E_{s}} \Theta_{s k}^{T} \xi_{s k} \operatorname{sgn}\left(s_{k}\right) \geq\left|\epsilon_{s k}\right|(27)\right. \\
\Theta_{M k}^{*}=\arg \min \left[\sup _{z_{s} \in E_{s}} \Theta_{M_{k}^{T}}^{T} \xi_{M_{k}} \operatorname{sgn}\left(s_{k}\right) \geq U_{M_{k}}\right](28)
\end{array}
$$

where $E_{s}$ is deined as follows:

$$
E_{s}=\left\{z_{s}: Z_{s_{j}}^{1} \leq z_{s j} \leq Z_{s_{j}}^{r_{s_{j}}}, j=1, \cdots, 3 n\right\}
$$

To simplify the problem, mild assumptions are given as $\|h\|_{\infty}<h^{U}(u),\|\Delta M\|_{\infty}<M^{U}\left(\mathbf{z}_{h}\right)$ and $\|\Delta C\|_{\infty}<$ $C_{s}^{U}\left(\mathrm{z}_{h}\right)$. Based on those assumptions, Proposition 3 is given.
Proposition 3 If the linguistic vector $\mathbf{z}_{h}$ falls into $\delta$ -box, $\Omega_{\alpha}\left(Z_{h}^{\alpha}, \delta\left(\mathbf{z}_{h}\right)\right)$, then the $k$-th element of optimal approximation error vector $\epsilon_{h}\left(\mathbf{z}_{h}\right)$ will be bounded by $\left|\epsilon_{h k}\right| \leq g_{k}\left(\mathbf{z}_{h}\right)^{T} \delta\left(\mathbf{z}_{h}\right)$, where $\epsilon_{h}\left(\mathbf{z}_{h}\right)=h-\Theta_{h}^{*} \otimes \xi_{h}, g_{k}$ is the $k$-th row vector of matrix $g\left(\mathbf{z}_{h}\right), g\left(\mathbf{z}_{h}\right) \in \Re^{n \times 2 n}$, and its element is defined as $g_{i j}=\sup _{\mathbf{z}_{h} \in \Omega_{\alpha}}\left[\left|\frac{\partial h_{i}\left(\mathbf{z}_{h}\right)}{\partial \mathbf{z}_{h j}}\right|\right]$ for $i=1, \cdots, n, j=1, \cdots, 2 n$

Now, let the control law be redesigned as

$$
\mathbf{f}=d(t)\left(\mathbf{f}_{h}+\mathbf{f}_{s}\right)+(1-d(t)) U_{s}\left(\mathbf{z}_{s}\right)+\widehat{\mathbf{f}},
$$

where

$$
d(t)=\left\{\begin{array}{ll}
1, & \text { as } \mathbf{z}_{s} \in E_{s} \\
0, & \text { otherwise. }
\end{array},\right.
$$

$$
U_{s}\left(\mathbf{z}_{s}\right)=\left(h^{U}\left(\mathbf{z}_{h}\right)+M^{U}\left\|\dot{\mathrm{x}}_{r}\right\|_{\infty}+C_{s}^{U}\|s\|_{\infty}\right) \operatorname{sgn}(\mathbf{s})
$$

Update laws are given as follows :

$$
\begin{array}{r}
\dot{\Theta}_{h}=r \xi_{h}\left(\mathbf{z}_{h}\right) \operatorname{diag}\left(\operatorname{sgn}\left(\mathbf{s}_{\Delta}\right)\right) \text { as } \mathbf{z}_{h} \in E \\
\dot{\Theta}_{s}=r \xi_{s}\left(\mathbf{z}_{s}\right) \operatorname{diag}\left(\operatorname{sgn}\left(\mathbf{s}_{\Delta}\right)\right) \text { as } \mathbf{z}_{s} \in E_{s} \\
\dot{\Theta}_{M}=r \xi_{M}\left(\mathbf{z}_{s}\right) \operatorname{diag}\left(\operatorname{sgn}\left(\mathbf{s}_{\Delta}\right)\right)\left\|\dot{x}_{r}\right\|_{\infty} \text { as } \mathbf{z}_{s} \in E_{s}
\end{array}
$$

for some $r>0$ and $\mathrm{s}_{\Delta}=\left[s_{\Delta_{1}}, \cdots, s_{\Delta_{k}}, \cdots, s_{\Delta_{n}}\right]^{T}$,

$$
\begin{aligned}
& s_{\Delta k}= \begin{cases}s_{k}-a_{k}, & \text { as } s_{k}<a_{k} ; \\
s_{k}-b_{k}, & \text { as } s_{k}>b_{k} ; \\
0, & \text { othewise. }\end{cases} \\
& \dot{s_{\Delta k}}=\dot{s}_{k}
\end{aligned}
$$

where $\mathbf{a}, \mathbf{b} \in \Re^{n}$ are some constant vectors and their the $k$-th elements satisfying $a_{k} \leq Z_{s_{2 n+k}}^{\alpha_{2 n+k}} \leq 0 \leq$ $Z_{s_{2 n+k}}^{\alpha_{2 n+k+1}} \leq b_{k}$ for someone index $\alpha_{2 n+k}$.

Theorem 1 If the control law and the update laws are given as in eq. (29) and in eq. (32)-(34), then tracking errors will asymptotically converge to a neighborhood of zero.

\section{Simulation and Experimental Re- sults}

A five degree-of-freedom (DOF) articulated robot arm is set up in the Intelligent Robot Laboratory of CS\&IE in NTU as shown Figure 2. A simulation is run in assumption that inertial matrix $M$, Coriolis, centrifugal torques $C$, and gravity vector $G$ of the arm are unknown. To show the effectiveness of adaptive robust law, $f_{s}$, we will neglect the compensation function $\mathbf{f}_{h}$. the position trajectories is given to move a cirle with period, 4 second and radius, $5 \mathrm{~cm}$ in $\mathbf{x}_{c}-\mathbf{y}_{c}$ plane, and in $z_{c}$ direction force trajectories is given as $f_{d 1}=10-10 \exp (-t) \mathrm{Nt}$. A simplified strategy for reducing memory-space usage is to decentralize the previous architecture and ignore $\dot{q}$, i.e. $\mathbf{f}_{s}\left(z_{s}\right)=f_{s}\left(x_{c}, s\right)$. The triangular form and sup-min operator are selected as membership functions and compositional operators. The total rule number of 
fuzzy controller is $5 \times 21 \times 21$ and initial parameter matrices of fuzzy rules are set to zeros. The real parameters are given as $k_{e}=10000(\mathrm{Nt} / \mathrm{m})$ and $x_{s}=5(\mathrm{~mm})$, and initial estimate values are given as $\widehat{k}_{e}(0)=5000$ and $\widehat{k_{e} x_{s}}(0)=0$. Simulation is run by 4-order RungeKutta method with fixed sampling time $0.5 \mathrm{~ms}$ and the force error $e_{f}$ result are listed in Figure 3. At the beginning, sinice initial parameter matrices are aet to zeros, which is similar to only use the PD controller to compensate for the uncertainties in the first period of circle motion, after the first period the tracking error is quickly driven toward zero. On the other hand, the virtual force error $e_{p}$ is given in the Fig. 4, we can find the error is fast converging to zero.

\section{Conclusions}

We had proposed a novel fuzzy hybrid force/position controller design method, which can update fuzzy rules to compensate unknown uncertainty of robot manipulator along with adaptive estimation of unknown parameters of task environment and guarantee the stability of the controlled robot manipulator systems. Besides, a dexterous use for fuzzy mathematics made the complicated scheme with computing efficiency. Present work is to implement this control algorithm to actual experiment.

\section{References}

[1] S. Chiaverini, L. Sciavicco "The Parallel Approach to Force/Position Control of Robot Manipulators" IEEE Transactions on Robotics and Automation vo. 9, no. 4, pp. 361-373, Aug. 1993

[2] R. Colbaugh, A. Engelmann "Adaptive Compliant Motion Control of Manipulators: Theory and Experiments" IEEE Robotics and Automation Conf, pp2719-2726, 1994

[3] G. Niemeyer, J-J E. Slotine, "Performance in Adaptive Manipulator Control ", The International Journal of Robotics Research, vol. 10, no. 2, $p p$. 149-161, 1991.

[4] K. Guglielmo, N. Sadegh "Implementating a Hybrid Learning Force Control Scheme "IEEE Control Systems vo. 14, no. 1, pp. 72-79 Feb. 1994

5] D. Jeon, M. Tomizuka "Learning Hybrid Force and Position Control of Robot Manipulators" IEEE Transactions on Robotics and Automation vo. 9, no. 4, pp. 423-432, Aug. 1993

[6] Sheng Liu and H. Asada, "Teaching and Learning of Deburring Robots Using Neural Networks" IEEE Conference on Robotics and Automation, pp. 339-345, 1993

[7] M. H. Raibert and J. J. Craig, "Hybrid Position/Force Control of Manipulators" ASME Jornal of Dynamic Systems, Measurement, and Control vol. $102 \mathrm{pp}$. 126-133, 1981
[8] Daniel E. Whitney, "Historical Perspective and State of the Art in Robot Force Control", The International Journal of Robotics Research, vol. 6, no. $1, p p .3-14,1987$.

[9] S. Sastry and M. Bodson, Adaptive Control: Stability, Convergence, and Robustness, Prentice Hall, pp.19 1989.

[10] J-J E. Slotine and W. Li, Applied Nonlinear Control, Englewood Cliffs, NJ: Prentice Hall, pp. 278284, 1991.

[11] J. Marsden, Elementary Classical Analysis. San Franciso: W. H. Freeman, 1974

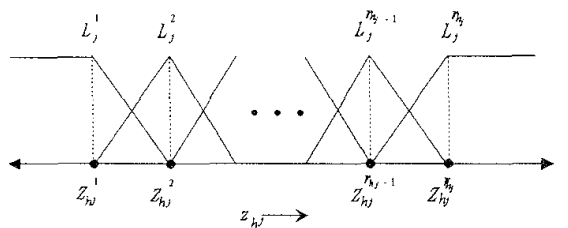

Fig. 1 The fuzzy enviroment of linguistic variable

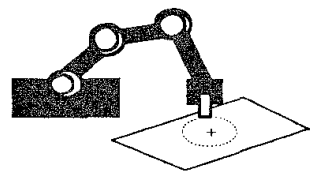

Fig. 2. The diagram of A-type robot

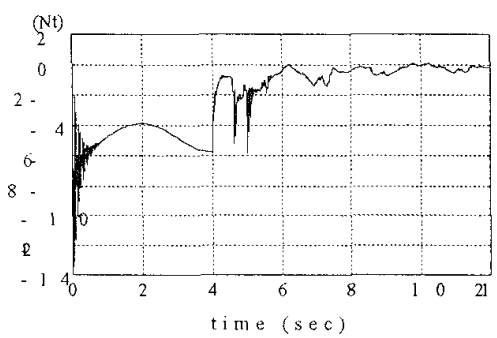

Fig. 3. Force error (Nt) V.S. time(sec)

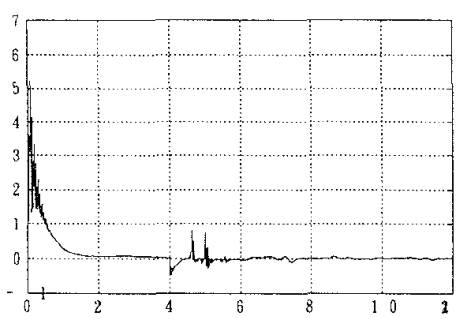

Fig. 4 Virtual force error (Nt) V.S. time(sec) 